\title{
Some unified formulas involving generalized-Apostol-type- Gould-Hopper polynomials and multiple power sums
}

\author{
Serkan Aracia, ${ }^{a, *}$ Mumtaz Riyasat ${ }^{b}$, Subuhi Khan ${ }^{b}$, Shahid Ahmad Wanib \\ ${ }^{a}$ Department of Economics, Faculty of Economics, Administrative and Social Sciences, Hasan Kalyoncu University, TR-27410 \\ Gaziantep, Turkey. \\ ${ }^{b}$ Department of Mathematics, Faculty of Science, Aligarh Muslim University, Aligarh, India.
}

\begin{abstract}
The article aims to introduce a new unified class of generalized Apostol type polynomials. Further, under a slight modification on the parameters associated to the generalized Apostol type and generalized Gould-Hopper polynomials and by the use of generating method, we introduce a new class of generalized-Apostol-type-Gould-Hopper polynomials. We state some quasi-monomial properties for a new class of extensions of generalized Apostol type polynomials as well as, some summation, multiplication and explicit formulae which connect this polynomial class with the $\lambda$-Stirling numbers of second kind and generalized Hurwitz zeta function. Some general symmetry identities involving multiple power sums are also established. The new class of polynomials contains as its special cases, not only the generalized-Gould-Hopper-Apostol-Bernoulli, Euler and Genocchi polynomials but many more known smaller classes of polynomials. Finally, the these polynomials are framed within the context of generalized modified Milne-Thomson's polynomials.
\end{abstract}

Keywords: Generalized-Apostol-type-Gould-Hopper polynomials, monomiality principle, summation formula, multiplication formulae, symmetry identities.

2010 MSC: 11B68, 05A10, 11B65.

(C)2019 All rights reserved.

\section{Introduction and preliminaries}

The theory of generalized special functions serves as an analytic foundation for the majority of problems in mathematical physics that have been solved exactly and finds broad practical applications. As an example, the generalized Hermite polynomials have been exploited to deal with quantum mechanical and optical beam transport problems and arise in problems ranging from the theory of partial differential equations to the abstract group theory $[8,9]$. We recall the following 2-variable forms of Hermite polynomials.

Definition 1.1. The 2-variable Hermite Kampé de Feriet polynomials (2VHKdFP) $H_{n}(x, y)$ [1] are defined

\footnotetext{
${ }^{*}$ Corresponding author

Email addresses: serkan.araci@hku.edu.tr (Serkan Araci), mumtazrst@gmail.com (Mumtaz Riyasat), subuhi2006@gmail.com (Subuhi Khan), shahidwani177@gmail .com (Shahid Ahmad Wani)
}

doi: 10.22436/jmcs.019.02.04

Received: 2017-08-21 Revised: 2018-06-03 Accepted: 2019-04-09 
by the following generating function:

$$
e^{x t+y t^{2}}=\sum_{n=0}^{\infty} H_{n}(x, y) \frac{t^{n}}{n !}
$$

Definition 1.2. The higher order Hermite polynomials or the Gould-Hopper polynomials $(G H P) H_{n}^{(j)}(x, y)$ are defined by the following generating function [11, p.58(6.3)]

$$
e^{x t+y t^{j}}=\sum_{n=0}^{\infty} H_{n}^{(j)}(x, y) \frac{t^{n}}{n !}, \quad j>2 .
$$

Definition 1.3. The generalized-Hermite polynomials (GHP) $H_{n}(x, y, c)$ are defined by the following generating function [37]:

$$
c^{x t+y t^{2}}=\sum_{n=0}^{\infty} H_{n}(x, y, c) \frac{t^{n}}{n !}, \quad c>1 .
$$

Definition 1.4. The generalized-Gould-Hopper polynomials (GGdHP) $H_{n}^{(j)}(x, y, c)$ are defined by the following generating function [41]:

$$
c^{x t+y t^{j}}=\sum_{n=0}^{\infty} H_{n}^{(j)}(x, y, c) \frac{t^{n}}{n !}, \quad c>1 ; \quad j>2 .
$$

These polynomials play an important role in problems involving Laplace's equation in parabolic coordinates, in quantum mechanics and in probability theory and are shown to be solutions of classical and generalized heat equations.

Operational methods provide powerful techniques to solve problems both in classical and quantum mechanics. Differential equations have been the primary motivation for the introduction of these techniques. The operational techniques combined with the monomiality principle [5] open new possibilities to deal with the theoretical foundations of special polynomials and also to introduce new families of special polynomials.

Definition 1.5. According to the monomiality principle, there exist two operators $\hat{M}$ and $\hat{P}$ playing, respectively, the role of multiplicative and derivative operators for a polynomial set $\left\{p_{\mathfrak{n}}(x)\right\}_{\mathfrak{n} \in \mathbb{N}}$, that is, $\hat{M}$ and $\hat{P}$ satisfy the following identities, for all $n \in \mathbb{N}$ :

$$
\hat{M}\left\{p_{n}(x)\right\}=p_{n+1}(x), \quad \hat{P}\left\{p_{n}(x)\right\}=n p_{n-1}(x) .
$$

The polynomial set $\left\{p_{\mathfrak{n}}(x)\right\}_{\mathfrak{n} \in \mathbb{N}}$ is then called a quasi-monomial. If $\hat{M}$ and $\hat{P}$ have differential realizations, then the polynomial set $\left\{p_{\mathfrak{n}}(x)\right\}_{\mathfrak{n} \in \mathbb{N}}$ satisfy the following differential equation:

$$
\hat{M} \hat{P}\left\{p_{\mathfrak{n}}(x)\right\}=n p_{\mathfrak{n}}(x) .
$$

Most of the properties of families of polynomials, recognized as quasi-monomial, can be deduced by using relevant multiplicative and derivative operators. The notion of quasi-monomiality has been exploited within different context. There is a continuous demand of solving problems by means of differential equations in research fields like classical and quantum optics. The aforementioned 2-variable forms of Hermite polynomials are all quasi-monomials and corresponding properties are derived using the rules associated with monomiality principle, for this see [6, 37, 41].

Various unified forms of Apostol-type polynomials, which are the unifications of the Apostol-Bernoulli, Euler and Genocchi polynomials are introduced and studied by many authors in a systematic manner, for this see $[2,3,13,19,23,24,26,28,29,34,35,38]$.

We recall the following generalized forms of the Apostol-Bernoulli, Euler and Genocchi polynomials introduced by many authors [14, 30, 31, 39]. 
Definition 1.6. Let $a, b, c \in \mathbb{R}^{+}$with $a \neq b, x \in \mathbb{R}$ and $n \in \mathbb{N}_{0}$. The generalized Apostol-Bernoulli polynomials $\mathfrak{B}_{\mathfrak{n}}^{(\alpha)}(x ; \lambda ; a, b, c)$ of order $\alpha \in \mathbb{Z}$ are defined by the following generating function [39]:

$$
\left(\frac{t}{\lambda b^{t}-a^{t}}\right)^{\alpha} c^{x t}=\sum_{n=0}^{\infty} \mathfrak{B}_{n}^{(\alpha)}(x ; a, b, c ; \lambda) \frac{t^{n}}{n !}, \quad|t|<\left|\frac{\ln (\lambda)}{\ln \left(\frac{a}{b}\right)}\right| .
$$

Definition 1.7. Let $a, b, c \in \mathbb{R}^{+}, x \in \mathbb{R}$ and $n \in \mathbb{N}_{0}$. The generalized Apostol-Euler polynomials $\mathfrak{E}_{n}^{(\alpha)}(x ; \lambda ; a, b, c)$ of order $\alpha \in \mathbb{Z}$ are defined by the following generating function:

$$
\left(\frac{2}{\lambda b^{t}+a^{t}}\right)^{\alpha} c^{x t}=\sum_{n=0}^{\infty} \mathfrak{E}_{n}^{(\alpha)}(x ; a, b, c ; \lambda) \frac{t^{n}}{n !}, \quad|t|<\left|\frac{\ln (-\lambda)}{\ln \left(\frac{a}{b}\right)}\right| .
$$

Definition 1.8. Let $a, b, c \in \mathbb{R}^{+}, x \in \mathbb{R}$ and $n \in \mathbb{N}_{0}$. The generalized Apostol-Genocchi polynomials $\mathcal{G}_{\mathrm{n}}^{(\alpha)}(x ; \lambda ; a, b, c)$ of order $\alpha \in \mathbb{Z}$ are defined by the following generating function [14]:

$$
\left(\frac{2 t}{\lambda b^{t}+a^{t}}\right)^{\alpha} c^{x t}=\sum_{n=0}^{\infty} \mathcal{G}_{n}^{(\alpha)}(x ; a, b, c ; \lambda) \frac{t^{n}}{n !}, \quad|t|<\left|\frac{\ln (-\lambda)}{\ln \left(\frac{a}{b}\right)}\right| .
$$

We note that

$$
\mathfrak{B}_{n}^{(\alpha)}(0 ; a, b, c ; \lambda):=\mathfrak{B}_{n}^{(\alpha)}(a, b, c ; \lambda), \mathfrak{E}_{n}^{(\alpha)}(0 ; a, b, c ; \lambda):=\mathfrak{E}_{n}^{(\alpha)}(a, b, c ; \lambda), \mathcal{G}_{n}^{(\alpha)}(0 ; a, b, c ; \lambda):=\mathcal{G}_{n}^{(\alpha)}(a, b, c ; \lambda),
$$

are the generalized Apostol-Bernoulli, Euler and Genocchi numbers, each of order $\alpha$.

For $b=c=e, a=1$, we have Apostol-Bernoulli, Euler and Genocchi polynomials, each of order $\alpha$, for this see $[25,27,32]$.

Here, we introduce the following unified form of the generalized Apostol-Bernoulli, Euler and Genocchi polynomials.

Definition 1.9. Let $a, b, c \in \mathbb{R}^{+}, x \in \mathbb{R} ; \lambda, \mu, v \in \mathbb{C}$ and $n \in \mathbb{N}_{0}$. The generalized Apostol type polynomials (GATP) $\mathcal{F}_{\mathfrak{n}}^{(\alpha)}(x ; a, b, c ; \mu, v ; \lambda)$ of order $\alpha \in \mathbb{Z}$ are defined by the following generating function:

$$
\left(\frac{2^{\mu} t^{v}}{\lambda b^{t}+a^{t}}\right)^{\alpha} c^{x t}=\sum_{n=0}^{\infty} \mathcal{F}_{n}^{(\alpha)}(x ; a, b, c ; \mu, v ; \lambda) \frac{t^{n}}{n !}, \quad|t|<\left|\frac{\ln (-\lambda)}{\ln \left(\frac{a}{b}\right)}\right|,
$$

where

$$
\mathcal{F}_{n}^{(\alpha)}(0 ; a, b, c ; \mu, v ; \lambda):=\mathcal{F}_{n}^{(\alpha)}(a, b, c ; \mu, v ; \lambda)
$$

denotes the generalized Apostol type numbers of order $\alpha$ defined by

$$
\left(\frac{2^{\mu} t^{v}}{\lambda b^{t}+a^{t}}\right)^{\alpha}=\sum_{n=0}^{\infty} \mathcal{F}_{n}^{(\alpha)}(a, b, c ; \mu, v ; \lambda) \frac{t^{n}}{n !}
$$

For $b=c=e, a=1$, we have Luo and Srivastava [34] Apostol-type polynomials $\mathcal{F}_{\mathfrak{n}}^{(\alpha)}(x ; \lambda ; \mu, v)$. In fact, from equations (1.4)-(1.6) and (1.7), we have

$$
\begin{aligned}
(-1)^{\alpha} \mathcal{F}_{n}^{(\alpha)}(x ; a, b, c ; 0,1 ;-\lambda) & =\mathfrak{B}_{n}^{(\alpha)}(x ; a, b, c ; \lambda), \\
\mathcal{F}_{n}^{(\alpha)}(x ; a, b, c ; 1,0 ; \lambda) & =\mathfrak{E}_{n}^{(\alpha)}(x ; a, b, c ; \lambda), \\
\mathcal{F}_{n}^{(\alpha)}(x ; a, b, c ; 1,1 ; \lambda) & =\mathcal{G}_{n}^{(\alpha)}(x ; a, b, c ; \lambda) .
\end{aligned}
$$

To introduce the hybridized forms of generalized Apostol type polynomials and to study their properties is a new investigation. The process of combining two multi-variable forms of special polynomials 
either by replacement technique or operational technique is called hybridization of polynomials, which shows that the properties of new hybridized polynomial lie within the context of parent polynomials. The multi-variable hybrid special polynomials are important as they possess important properties such as recurrence and explicit relations and functional and differential equations, summation formulae, symmetric and convolution identities etc.. The usefulness and potential for applications of various properties of multi-variable hybrid special polynomials in certain problems of number theory, combinatorics, classical and numerical analysis, theoretical physics, approximation theory and other fields of pure and applied mathematics arose the desire to introduce a new hybrid class of generalized-Apostol-type-Gould-Hopper polynomials.

In this article, a hybrid class of generalized-Apostol-type-Gould-Hopper polynomials is introduced and their properties are being characterized via different generating functions method. The differential operators, differential equations, summation, multiplication and explicit formula and symmetry identities for these polynomials are established. Certain illustrative special cases are considered and analogous properties for these special cases are deduced.

\section{Generalized-Apostol-type-Gould-Hopper polynomials}

In this section, a hybrid class of generalized-Apostol-type-Gould-Hopper polynomials (GATGdHP) is introduced by convoluting GGdHP and GATP. The GGdHATP are defined as discrete generalized GouldHopper convolution of generalized-Apostol type polynomials.

First, we introduce the generating function applying replacement technique for the generalizedApostol-type-Gould-Hopper polynomials. For this, the following result is proved.

Theorem 2.1. Let $\mathrm{a}, \mathrm{b}, \mathrm{c} \in \mathbb{R}^{+}, x, y \in \mathbb{R} ; \lambda, \mu, v \in \mathbb{C}$ and $\mathrm{n} \in \mathbb{N}_{0}$. The generalized-Apostol-type-GouldHopper polynomials are defined by the following generating function:

$$
\left(\frac{2^{\mu} t^{\nu}}{\lambda b^{t}+a^{t}}\right)^{\alpha} \quad c^{x t+y t^{j}}=\sum_{n=0}^{\infty} H_{n} \mathcal{F}_{n}^{(\alpha, j)}(x, y ; a, b, c ; \mu, v ; \lambda) \frac{t^{n}}{n !}, \quad c>1 ; j>2 ;|t|<\left|\frac{\ln (-\lambda)}{\ln \left(\frac{a}{b}\right)}\right| .
$$

Proof. Expanding the exponential function $e^{(x \log c) t}$ and then replacing the powers of $x$, i.e., $(x \log c)^{0}$, $(x \log c)^{1},(x \log c)^{2}, \ldots,(x \log c)^{n}$ by the polynomials $H_{0}^{(j)}(x, y, c), H_{1}^{(j)}(x, y, c), \ldots, H_{n}^{(j)}(x, y, c)$ in the l.h.s. and $x$ by the polynomial $H_{1}^{(j)}(x, y, c)$ in the r.h.s. of equation (1.7) and after summing up the terms in the 1.h.s. of the resultant equation, we have

$$
\left(\frac{2^{\mu} t^{v}}{\lambda b^{t}+a^{t}}\right)^{\alpha} \sum_{n=0}^{\infty} H_{n}^{(j)}(x, y, c) \frac{t^{n}}{n !}=\sum_{n=0}^{\infty} \mathcal{F}_{n}^{(\alpha)}\left(\left(H_{1}^{(j)}(x, y, c) ; a, b, c ; \mu, v ; \lambda\right)\right) \frac{t^{n}}{n !},
$$

which on using equation (1.1) in the l.h.s. and denoting the resultant GATGdHP in the r.h.s. by ${ }_{H} \mathcal{F}_{\mathrm{n}}^{(\alpha, j)}(x, y ; a, b, c ; \mu, v ; \lambda)$, we are led to assertion (2.1).

Corollary 2.2. Let $a, b, c \in \mathbb{R}^{+}, x, y \in \mathbb{R} ; \lambda, \mu, v \in \mathbb{C} ; n \in \mathbb{N}_{0}$ and $j=2$. The generalized-Apostol-typeGould-Hopper polynomials are defined by the following generating function:

$$
\left(\frac{2^{\mu} t^{\nu}}{\lambda b^{t}+a^{t}}\right)^{\alpha} c^{x t+y t^{2}}=\sum_{n=0}^{\infty} H^{\mathcal{F}_{n}^{(\alpha)}}(x, y ; a, b, c ; \mu, v ; \lambda) \frac{t^{n}}{n !}, \quad c>1 ;|t|<\left|\frac{\ln (-\lambda)}{\ln \left(\frac{a}{b}\right)}\right| .
$$

Note 1. For $b=c=e, a=1$, equations (2.1) and (2.2) yield the Apostol-type-Gould-Hopper polynomials ${ }_{H} \mathcal{F}_{n}^{(\alpha, j)}(x, y ; \mu, v ; \lambda)$ and Apostol-type-Hermite polynomials ${ }_{H} \mathcal{F}_{n}^{(\alpha)}(x, y ; \mu, v ; \lambda)$, which for $x \rightarrow 2 x, y=-1$ becomes the Apostol-type-Hermite polynomials ${ }_{H} \mathcal{F}_{n}^{(\alpha)}(x ; \mu, v ; \lambda)$, for these polynomials see [19]. 
Remark 2.3. For the special case $\lambda \rightarrow-\lambda ; \mu=0$ and $v=1$ and on use of $(-1)^{\alpha}{ }_{H} \mathcal{F}_{n}^{(\alpha, j)}(x, y ; a, b, c ; 0,1 ; \lambda)=$ $\mathrm{H}^{\mathfrak{B}_{n}^{(\alpha, j)}}(x, y ; a, b, c ; \lambda)$, we have generalized-Gould-Hopper-Apostol-Bernoulli polynomials defined by

$$
\left(\frac{t}{\lambda b^{t}-a^{t}}\right)^{\alpha} c^{x t+y t^{j}}=\sum_{n=0}^{\infty} H^{B_{n}^{(\alpha, j)}}(x, y ; a, b, c ; \mu, v ; \lambda) \frac{t^{n}}{n !}, \quad c>1 ; j>2 ; \quad|t|<\left|\frac{\ln (\lambda)}{\ln \left(\frac{a}{b}\right)}\right| .
$$

Remark 2.4. For the special case $\mu=1$ and $v=0$ and on use of ${ }_{H} \mathcal{F}_{n}^{(\alpha, j)}(x, y ; a, b, c ; 1,0 ; \lambda)={ }_{H} \mathfrak{E}_{n}^{(\alpha, j)}(x, y$; $a, b, c ; \lambda)$, we have generalized-Gould-Hopper-Apostol-Euler polynomials defined by

$$
\left(\frac{2}{\lambda b^{t}+a^{t}}\right)^{\alpha} c^{x t+y t^{j}}=\sum_{n=0}^{\infty} H \mathfrak{E}_{n}^{(\alpha, j)}(x, y ; a, b, c ; \mu, v ; \lambda) \frac{t^{n}}{n !}, \quad c>1 ; j>2 ;|t|<\left|\frac{\ln (-\lambda)}{\ln \left(\frac{a}{b}\right)}\right| .
$$

Remark 2.5. For the special case $\mu=1$ and $v=1$ and on use of ${ }_{H} \mathcal{F}_{n}^{(\alpha, j)}(x, y ; a, b, c ; 1,1 ; \lambda)={ }_{H} \mathcal{G}_{n}^{(\alpha, j)}(x, y$; $a, b, c ; \lambda)$, we have generalized-Gould-Hopper-Apostol-Genocchi polynomials defined by

$$
\left(\frac{2 t}{\lambda b^{t}+a^{t}}\right)^{\alpha} c^{x t+y t^{j}}=\sum_{n=0}^{\infty} H \mathcal{G}_{n}^{(\alpha, j)}(x, y ; a, b, c ; \mu, v ; \lambda) \frac{t^{n}}{n !}, \quad c>1 ; j>2 ; \quad|t|<\left|\frac{\ln (-\lambda)}{\ln \left(\frac{a}{b}\right)}\right| .
$$

Note 2. For $\mathrm{b}=\mathrm{c}=e, \mathrm{a}=1$, Remarks 2.3-2.5 give the Gould-Hopper-Apostol-Bernoulli, Euler and Genocchi polynomials, each of order $\alpha$, which for $\lambda=1$ yield Gould-Hopper-Bernoulli, Euler and Genocchi polynomials, each of order $\alpha$, which again for $\alpha=1$ yield Gould-Hopper-Bernoulli, Euler and Genocchi polynomials [17, 19].

Note 3. For $j=2$, Remarks 2.3-2.5 give the generalized-Hermite-Apostol-Bernoulli, Euler and Genocchi polynomials, each of order $\alpha$, which for $b=c=e, a=1$ yield Hermite-Apostol-Bernoulli, Euler and Genocchi polynomials, each of order $\alpha$, which again for $\lambda=1$ yield Hermite-Bernoulli, Euler and Genocchi polynomials, each of order $\alpha$, which further for $\alpha=1$ reduce to Hermite-Bernoulli, Euler and Genocchi polynomials. Some of these polynomials are given in $[16,37]$.

Theorem 2.6. The generalized-Apostol-type-Gould-Hopper polynomials are defined by the following series expansion:

$$
H^{\mathcal{F}_{n}^{(\alpha, j)}}(x, y ; a, b, c ; \mu, v ; \lambda)=n ! \sum_{k=0}^{n} \sum_{s=0}^{\left[\frac{k}{j}\right]} \frac{\mathcal{F}_{n-k}^{(\alpha)}(a, b, c ; \mu, v ; \lambda) x^{k-j s} y^{s}(\log c)^{k+s-j s}}{(n-k) !(k-j s) ! s !} .
$$

Proof. Using equations (1.1) and (1.8) in generating function (2.1) yields

$$
\sum_{n=0}^{\infty} H_{\mathcal{F}_{n}^{(\alpha, j)}}(x, y ; a, b, c ; \mu, v ; \lambda) \frac{\mathrm{t}^{\mathrm{n}}}{n !}=\sum_{n=0}^{\infty} \mathcal{F}_{n}^{(\alpha)}(a, b, c ; \mu, v ; \lambda) \frac{\mathrm{t}^{\mathrm{n}}}{\mathrm{n} !} \sum_{k=0}^{\infty} H_{k}^{(j)}(x, y ; c) \frac{\mathrm{t}^{\mathrm{k}}}{k !},
$$

which on using the series expansion of generalized Gould-Hopper polynomials from [41] gives

$$
\sum_{n=0}^{\infty} H_{\mathcal{F}_{n}^{(\alpha, j)}}(x, y ; a, b, c ; \mu, v ; \lambda) \frac{t^{n}}{n !}=\sum_{n=0}^{\infty} \mathcal{F}_{n}^{(\alpha)}(a, b, c ; \mu, v ; \lambda) \frac{t^{n}}{n !} \sum_{k=0}^{\infty} k ! \sum_{s=0}^{\left[\frac{k}{j}\right]} \frac{x^{k-j s} y^{s}(\log c)^{k+s-j s}}{(k-j s) ! s !} \frac{t^{k}}{k !}
$$

On applying Cauchy product rule in above equation, we find

$$
\sum_{n=0}^{\infty} H_{\mathcal{F}_{n}^{(\alpha, j)}}(x, y ; a, b, c ; \mu, v ; \lambda) \frac{\mathrm{t}^{n}}{n !}=\sum_{n=0}^{\infty} n ! \sum_{k=0}^{n} \sum_{s=0}^{\left[\frac{k}{j}\right]} \frac{\mathcal{F}_{n-k}^{(\alpha)}(a, b, c ; \mu, v ; \lambda) x^{k-j s} y^{s}(\log c)^{k+s-j s}}{(n-k) !(k-j s) ! s !} \frac{t^{n}}{n !}
$$

which on equating the coefficients of same powers of $t$ yields assertion (2.2). 
In order to show that ATGGdHP are quasi-monomial, we prove the following result.

Theorem 2.7. The generalized-Apostol-type-Gould-Hopper polynomials are quasi-monomial with respect to the following multiplicative and derivative operators:

$$
\begin{aligned}
M_{H^{\mathcal{F}(\alpha, j)}}:= & \left(x \log c+j y(\log c)^{2-j} \partial_{\chi}^{j-1}\right) \\
& +\frac{\alpha v\left(\lambda b^{(\log c)^{-1} \partial_{x}}+a^{(\log c)^{-1} \partial_{x}}\right)-\alpha\left(\lambda b^{(\log c)^{-1} \partial_{x}} \log b+a^{(\log c)^{-1} \partial_{x}} \log a\right)}{(\log c)^{-1} \partial_{x}\left(\lambda b^{(\log c)^{-1} \partial_{x}}+a^{(\log c)^{-1} \partial_{x}}\right)}, \\
P_{H^{F}(\alpha, j)}:= & (\log c)^{-1} \partial_{x} .
\end{aligned}
$$

Proof. On differentiation of equation (2.1) with respect to $x$, we obtain the following identity:

$$
(\log c)^{-1} \partial_{x}\left\{\left(\frac{2^{\mu} t^{v}}{\lambda b^{t}+a^{t}}\right)^{\alpha} c^{x t+y t^{j}}\right\}=t\left(\frac{2^{\mu} t^{v}}{\lambda b^{t}+a^{t}}\right)^{\alpha} c^{x t+y t^{j}} .
$$

Again, differentiating both sides of the equation (2.1) w.r.t. $t$, we find

$$
\begin{aligned}
& \left(\left(x+j y t^{j-1}\right) \log c+\frac{\alpha v\left(\lambda b^{t}+a^{t}\right)-\alpha\left(\lambda b^{t} \log b+a^{t} \log a\right)}{t\left(\lambda b^{t}+a^{t}\right)}\right)\left(\frac{2^{\mu} t^{v}}{\lambda b^{t}+a^{t}}\right)^{\alpha} c^{x t+y t^{j}}
\end{aligned}
$$

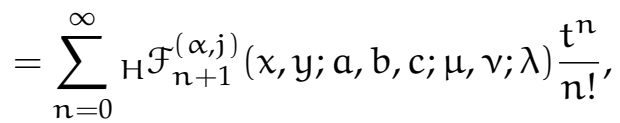

which on using identity (2.5) and equation (2.1) becomes

$$
\begin{aligned}
& \left(\left(x \log c+j y(\log c)^{2-j} \partial_{x}^{j-1}\right)\right. \\
& \left.+\frac{\alpha v\left(\lambda b^{(\log c)^{-1} \partial_{x}}+a^{(\log c)^{-1} \partial_{x}}\right)-\alpha\left(\lambda b^{(\log c)^{-1} \partial_{x}} \log b+a^{(\log c)^{-1} \partial_{x}} \log a\right)}{(\log c)^{-1} \partial_{x}\left(\lambda b^{(\log c)^{-1} \partial_{x}}+a^{(\log c)^{-1} \partial_{x}}\right)}\right) \\
& \times \sum_{n=0}^{\infty} H_{\mathcal{F}_{n}^{(\alpha, j)}}(x, y ; a, b, c ; \mu, v ; \lambda) \frac{\mathrm{t}^{n}}{n !}=\sum_{n=0}^{\infty} H_{\mathcal{F}^{(\alpha, j)}}(x, y ; a, b, c ; \mu, v ; \lambda) \frac{\mathrm{t}^{\mathrm{n}}}{n !} .
\end{aligned}
$$

On equating the coefficients of same power of $t$ in both sides of the above equation and in view of monomiality principle equation (1.2) yields assertion (2.3).

Using equation (2.1) in identity (2.5) yields

$$
(\log c)^{-1} \partial_{x}\left\{\sum_{n=0}^{\infty} H_{\mathcal{F}_{n}^{(\alpha, j)}}(x, y ; a, b, c ; \mu, v ; \lambda) \frac{t^{n}}{n !}\right\}=\sum_{n=0}^{\infty} H^{\mathcal{F}_{n-1}^{(\alpha, j)}}(x, y ; a, b, c ; \mu, v ; \lambda) \frac{t^{n}}{n !} .
$$

On equating the coefficients of same power of $t$ in both sides of the above equation and in view of monomiality principle equation (1.2) yields assertion (2.4).

To derive the differential equation for the ATGGdHP, the following result is proved.

Theorem 2.8. The generalized-Apostol-type-Gould-Hopper polynomials satisfy the following differential equation:

$$
\begin{aligned}
& \left(x \partial_{x}+j y(\log c)^{1-j} \partial_{x}^{j}+\frac{\alpha v\left(\lambda b^{(\log c)^{-1}} \partial_{x}+a^{(\log c)^{-1} \partial_{x}}\right)-\alpha\left(\lambda b^{(\log c)^{-1} \partial_{x}} \log b+a^{(\log c)^{-1} \partial_{x}} \log a\right)}{\left(\lambda b^{(\log c)^{-1} \partial_{x}}+a^{(\log c)^{-1} \partial_{x}}\right)}\right. \\
& \left.-n(\log c)^{-1} \partial_{x}\right){ }_{H} \mathcal{F}_{n}^{(\alpha, j)}(x, y ; a, b, c ; \mu, v ; \lambda)=0 \text {. }
\end{aligned}
$$


Proof. Using of expressions of multiplicative and derivative operators given by equations (2.3) and (2.4) in equation (1.3) and on simplification, we get the required differential equation given by (2.7).

The series expansions, differential operators and differential equations for the above special cases and particular members can be easily obtained by choosing suitable values of parameters. Thus we omit them.

In the next section, we gave some unified formula for the generalized-Apostol-type-Gould-Hopper polynomials.

\section{Summation, multiplication and explicit formula}

There is a continuous demand of solving problems by means of formulas, functions equations, relations and identities in research fields like classical and quantum optics. These formulas, functional equations and identities arise in well-defined combinatorial contexts and they lead systematically to welldefined classes of functions. It happens very often that the solution of a given problem in physics or applied mathematics requires the evaluation of infinite sums involving special polynomials. The summation formula of hybrid type special polynomials of more than one variable often appear in applications ranging from electromagnetic processes to combinatorics, see for example [7, 15]. This provides motivation to establish some unified formula for the hybrid type generalized-Apostol-type-Gould-Hopper polynomials.

First, we establish some summation formula for the generalized-Apostol-type-Gould-Hopper polynomials. For this we prove the following theorems.

Theorem 3.1. For $\alpha, \beta \in Z$, the following relation for the generalized-Apostol-type-Gould-Hopper polynomials holds true:

$$
H^{\mathcal{F}_{n}^{(\alpha \pm \beta, j)}}(x, y ; a, b, c ; \mu, v ; \lambda)=\sum_{k=0}^{n}\left(\begin{array}{l}
n \\
k
\end{array}\right) H_{\mathcal{F}^{-k}}^{(\alpha, j)}(x, y ; a, b, c ; \mu, v ; \lambda) \mathcal{F}_{k}^{( \pm \beta)}(a, b, c ; \mu, v ; \lambda) .
$$

Proof. We write generating function (2.1) in the following form:

$$
\left(\frac{2^{\mu} t^{v}}{\lambda b^{t}+a^{t}}\right)^{\alpha \pm \beta} c^{x t+y t^{j}}=\sum_{n=0}^{\infty} H_{n} \mathcal{F}_{n}^{(\alpha \pm \beta, j)}(x, y ; a, b, c ; \mu, v ; \lambda) \frac{t^{n}}{n !},
$$

which on using equations (1.8) and (2.1) becomes

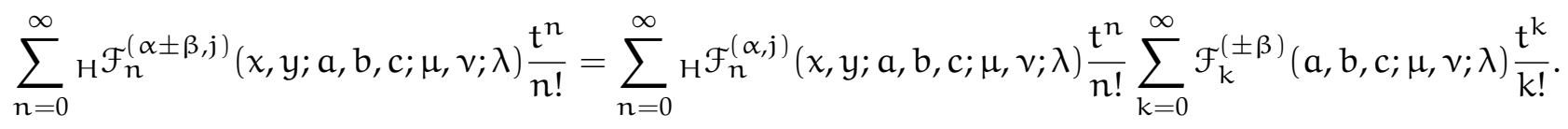

Using Cauchy product rule in above equation and then equating the coefficients of same powers of $t$ in both sides of resultant equation yields assertion (3.1).

Theorem 3.2. The following implicit summation formula for the generalized-Apostol-type-Gould-Hopper polynomials holds true:

$$
{ }_{H} \mathcal{F}_{n}^{(\alpha, j)}(x+w, y ; a, b, c ; \mu, v ; \lambda)=\sum_{k=0}^{n}\left(\begin{array}{l}
n \\
k
\end{array}\right) w^{k}(\log c)^{k}{ }_{H} \mathcal{F}_{n-k}^{(\alpha, j)}(x, y ; a, b, c ; \mu, v ; \lambda) .
$$

Proof. Replacing $x$ by $x+w$ in generating relation (2.1), we have

$$
\left(\frac{2^{\mu} t^{v}}{\lambda b^{t}+a^{t}}\right)^{\alpha} c^{(x+w) t+y t^{j}}=\sum_{n=0}^{\infty} H_{n}^{\mathcal{F}_{n}^{(\alpha, j)}}(x+w, y ; a, b, c ; \mu, v ; \lambda) \frac{t^{n}}{n !},
$$


which can be written as

$$
\left(\frac{2^{\mu} t^{v}}{\lambda b^{t}+a^{t}}\right)^{\alpha} c^{x t+y t^{j}} \exp (\log c w t)=\sum_{n=0}^{\infty} H_{\mathcal{F}_{n}^{(\alpha, j)}}(x+w, y ; a, b, c ; \mu, v ; \lambda) \frac{t^{n}}{n !} .
$$

Using the generating function equation (2.1) and expanding the exponential in the 1.h.s. of above the equation, we have

$$
\left(\sum_{n=0}^{\infty} H^{\mathcal{F}_{n}^{(\alpha, j)}}(x, y ; a, b, c ; \mu, v ; \lambda) \frac{t^{n}}{n !}\right)\left(\sum_{k=0}^{\infty} w^{k}(\log c)^{k} \frac{t^{k}}{k !}\right)=\sum_{n=0}^{\infty} H^{\mathcal{F}_{n}^{(\alpha, j)}}(x+w, y ; a, b, c ; \mu, v ; \lambda) \frac{t^{n}}{n !},
$$

which on using Cauchy product rule in the l.h.s. and then equating the coefficients of like powers of $t$ on both sides yields assertion (3.2).

Corollary 3.3. For $w=1$ in equation (3.2), we have following implicit summation formula for the generalizedApostol-type-Gould-Hopper polynomials:

$$
{ }_{H} \mathcal{F}_{n}^{(\alpha, j)}(x+1, y ; a, b, c ; \mu, v ; \lambda)=\sum_{k=0}^{n}\left(\begin{array}{l}
n \\
k
\end{array}\right)(\log c)^{k}{ }_{H} \mathcal{F}_{n-k}^{(\alpha, j)}(x, y ; a, b, c ; \mu, v ; \lambda) .
$$

Theorem 3.4. The following implicit summation formula for the generalized-Apostol-type-Gould-Hopper polynomials holds true:

$$
{ }_{H} \mathcal{F}_{n+m}^{(\alpha, j)}(z, y ; a, b, c ; \mu, v ; \lambda)=\sum_{s, k=0}^{m, n}\left(\begin{array}{c}
m \\
s
\end{array}\right)\left(\begin{array}{l}
n \\
k
\end{array}\right)(\log c)^{s+k}(z-x)^{s+k}{ }_{H} \mathcal{F}_{n+m-s-k}^{(\alpha, j)}(x, y ; a, b, c ; \mu, v ; \lambda) .
$$

Proof. Replacing $t \rightarrow t+u$ in generating function (2.1) and using the following rule:

$$
\sum_{N=0}^{\infty} f(N) \frac{(x+y)^{N}}{N !}=\sum_{l, m=0}^{\infty} f(l+m) \frac{x^{l} y^{m}}{l ! m !}
$$

in the r.h.s. of the resultant equation, we find

$$
\left(\frac{2^{\mu}(t+u)^{v}}{\lambda b^{t+u}+a^{t+u}}\right)^{\alpha} c^{x(t+u)+y(t+u)^{j}}=\sum_{n, m=0}^{\infty} H^{F_{n+m}^{(\alpha, j)}}(x, y ; a, b, c ; \mu, v ; \lambda) \frac{t^{n}}{n !} \frac{u^{m}}{m !} .
$$

Rewriting equation (3.5) as:

$$
\left(\frac{2^{\mu}(t+u)^{v}}{\lambda b^{t+u}+a^{t+u}}\right)^{\alpha} c^{y(t+u)^{j}}=c^{-x(t+u)} \sum_{n, m=0}^{\infty} H^{F_{n+m}^{(\alpha, j)}}(x, y ; a, b, c ; \mu, v ; \lambda) \frac{t^{n}}{n !} \frac{u^{m}}{m !} .
$$

Replacing $x$ by $z$ in the above equation and then equating the resultant equation to the above equation, we find

$$
\sum_{n, m=0}^{\infty} H_{\mathcal{F}_{n+m}^{(\alpha, j)}}(z, y ; a, b, c ; \mu, v ; \lambda) \frac{t^{n}}{n !} \frac{u^{m}}{m !}=c^{(z-x)(t+u)} \sum_{n, m=0}^{\infty} H_{\mathcal{F}_{n+m}^{(\alpha, j)}}(x, y ; a, b, c ; \mu, v ; \lambda) \frac{t^{n}}{n !} \frac{u^{m}}{m !},
$$

which on writing $c^{(z-x)(t+u)}$ in the form of exponential and then expanding the exponential in the r.h.s. gives

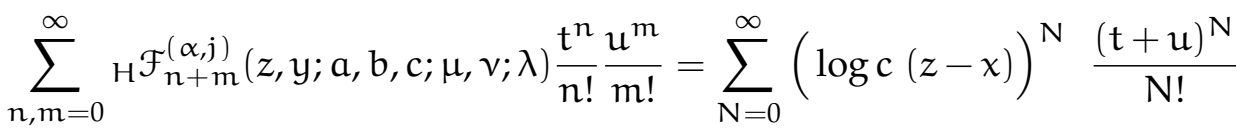

$$
\begin{aligned}
& \times \sum_{n, m=0}^{\infty} H^{\mathcal{F}_{n+m}^{(\alpha, j)}}(x, y ; a, b, c ; \mu, v ; \lambda) \frac{t^{n}}{n !} \frac{u^{m}}{m !} \text {. }
\end{aligned}
$$


Again, on use of equation (3.4) in the r.h.s. of equation (3.6), we find

$$
\begin{aligned}
& \sum_{n, m=0}^{\infty} H_{\mathcal{F}_{n+m}^{(\alpha, j)}}(z, y ; a, b, c ; \mu, v ; \lambda) \frac{t^{n}}{n !} \frac{u^{m}}{m !}=\sum_{k, s=0}^{\infty}(\log c)^{s+k}(z-x)^{s+k} \frac{t^{k}}{k !} \frac{u^{s}}{s !} \\
& \times \sum_{n, m=0}^{\infty} H_{\mathcal{F}_{n+m}^{(\alpha, j)}}(x, y ; a, b, c ; \mu, v ; \lambda) \frac{\mathrm{t}^{n}}{n !} \frac{u^{m}}{m !} .
\end{aligned}
$$

Now, replacing $n \rightarrow n-k$ and $m \rightarrow m-s$ in the r.h.s. of equation (3.7), we find

$$
\begin{aligned}
\sum_{n, m=0}^{\infty} H_{\mathcal{F}^{(\alpha, j)}}^{(\alpha+m}(z, y ; a, b, c ; \mu, v ; \lambda) \frac{t^{n}}{n !} \frac{u^{m}}{m !}= & \sum_{n, m=0}^{\infty} \sum_{k, s=0}^{n, m}(\log c)^{s+k} \frac{(z-x)^{s+k}}{k ! s !} \\
& \times{ }_{H} \mathcal{F}_{n+m-s-k}^{(\alpha, j)}(x, y ; a, b, c ; \mu, v ; \lambda) \frac{t^{n}}{(n-k) !} \frac{u^{m}}{(m-s) !} .
\end{aligned}
$$

Equating the coefficients of the same powers of $t$ and $u$ in both sides of equation (3.8), we are led to assertion (3.3).

Theorem 3.5. Let $\mathrm{a}, \mathrm{b}$, and $\mathrm{c}$ be positive integers by $\mathrm{a} \neq \mathrm{b}$. Then for $\mathrm{x}, \mathrm{y} \in \mathbb{R}$ and $\mathrm{n} \geqslant 0$, we have

$$
{ }_{H} \mathcal{F}_{n}^{(\alpha, j)}(x+\alpha, y ; a, b, c ; \mu, v ; \lambda)=n ! \sum_{k=0}^{\left[\frac{n}{j}\right]} \frac{y^{k}(\log c)^{k}{ }_{H^{\prime}} \mathcal{F}_{n-j k}^{(\alpha)}\left(x ; \frac{a}{c}, \frac{b}{c}, c ; \mu, v ; \lambda\right)}{(n-j k) ! k !} .
$$

Proof. Replacing $x$ by $x+\alpha$ in generating relation (2.1), we have

$$
\sum_{n=0}^{\infty} H_{\mathcal{F}_{n}^{(\alpha, j)}}(x+\alpha, y ; a, b, c ; \mu, v ; \lambda) \frac{t^{n}}{n !}=\left(\frac{2^{\mu} t^{\nu}}{\lambda b^{t}+a^{t}}\right)^{\alpha} c^{(x+\alpha) t+y t^{j}}
$$

which can be written as

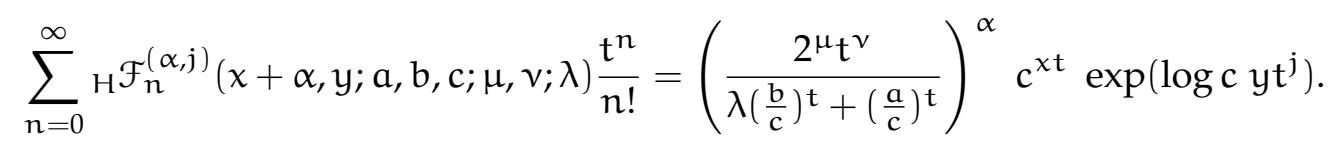

Now, using the generating equation (1.7) and expanding the exponential in the r.h.s. of above the equation, we have

$$
\sum_{n=0}^{\infty} H_{H_{n}} \mathcal{F}^{(\alpha, j)}(x+\alpha, y ; a, b, c ; \mu, v ; \lambda) \frac{t^{n}}{n !}=\left(\sum_{n=0}^{\infty} \mathcal{F}_{n}^{(\alpha)}\left(x ; \frac{a}{c}, \frac{b}{c}, c ; \mu, v ; \lambda\right) \frac{t^{n}}{n !}\right)\left(\sum_{k=0}^{\infty} y^{k}(\log c)^{k} \frac{t^{j k}}{k !}\right),
$$

which on applying Cauchy product rule in the 1.h.s. and then equating the coefficients of like powers of $t$ in both sides yields assertion (3.9).

Theorem 3.6. Let $\mathrm{a}, \mathrm{b}$ and $\mathrm{c}$ be positive integers by $\mathrm{a} \neq \mathrm{b}$. Then for $\mathrm{x}, \mathrm{y} \in \mathbb{R}$ and $\mathrm{n} \geqslant 0$, we have

$$
H^{\mathcal{F}_{n}^{(\alpha, j)}}(x, y ; a, b, c ; \mu, v ; \lambda)=\sum_{k=0}^{n}\left(\begin{array}{l}
n \\
k
\end{array}\right) \mathcal{F}_{n-k}^{(\alpha)}(a, b, c ; \mu, v ; \lambda) H_{k}^{(j)}(x, y, c) .
$$

Proof. By use of equations (1.8) and (1.1), we have

$$
\left(\frac{2^{\mu} t^{v}}{\lambda b^{t}+a^{t}}\right)^{\alpha} c^{x t+y t^{j}}=\left(\sum_{n=0}^{\infty} \mathcal{F}_{n}^{(\alpha)}(a, b, c ; \mu, v ; \lambda) \frac{t^{n}}{n !}\right)\left(\sum_{k=0}^{\infty} H_{k}^{(j)}(x, y, c) \frac{t^{k}}{k !}\right) .
$$

Using Cauchy product rule in the r.h.s. of above equation and then equating the coefficients of like powers of $t$ on both sides of the resultant equation, yields assertion (3.10). 
Theorem 3.7. Let $\mathrm{a}, \mathrm{b}$, and $\mathrm{c}$ be positive integers by $\mathrm{a} \neq \mathrm{b}$. Then for $\mathrm{x}, \mathrm{y} \in \mathbb{R}$ and $\mathrm{n} \geqslant 0$, we have

$$
{ }_{H} \mathcal{F}_{k}^{(\alpha, j)}(x, y ; a, b, c ; \mu, v ; \lambda)=k ! \sum_{s=0}^{\left[\frac{k}{j}\right]} \sum_{n=0}^{k-s j} \frac{x^{k-s j-n}(\log c)^{k-s(j-1)-n} y^{s}}{(k-s j-n) ! s ! n !} \mathcal{F}_{n}^{(\alpha)}(a, b, c ; \mu, v ; \lambda)
$$

Proof. By the use of equation (1.8) and expansions of $e^{(\log c) x t}$ and $e^{(\log c) y t^{j}}$ in generating equation (2.1), we have

$$
\sum_{n=0}^{\infty} H_{\mathcal{F}_{n}^{(\alpha, j)}}(x, y ; a, b, c ; \mu, v ; \lambda) \frac{t^{n}}{n !}=\sum_{n=0}^{\infty} \mathcal{F}_{n}^{(\alpha)}(a, b, c ; \mu, v ; \lambda) \frac{t^{n}}{n !} \sum_{k=0}^{\infty} x^{k}(\log c)^{k} \frac{t^{k}}{k !} \sum_{s=0}^{\infty} y^{s}(\log c)^{s} \frac{t^{s j}}{s !},
$$

which on applying Cauchy product rule becomes

$$
\begin{aligned}
& \sum_{k=0}^{\infty} H^{\mathcal{F}_{k}^{(\alpha, j)}}(x, y ; a, b, c ; \mu, v ; \lambda) \frac{t^{k}}{k !}=\sum_{k=0}^{\infty}\left(\sum_{n=0}^{k}\left(\begin{array}{l}
k \\
n
\end{array}\right) \mathcal{F}_{n}^{(\alpha)}(a, b, c ; \mu, v ; \lambda) x^{k-n}(\log c)^{k-n}\right) \frac{t^{k}}{k !} \times \sum_{s=0}^{\infty} y^{s}(\log c)^{s} \frac{t^{s j}}{s !} \\
& =\sum_{k=0}^{\infty}\left(\sum_{s=0}^{\left[\frac{k}{j}\right]} \sum_{n=0}^{k-s j}\left(\begin{array}{c}
k-s j \\
n
\end{array}\right) \mathcal{F}_{n}^{(\alpha)}(a, b, c ; \mu, v ; \lambda) x^{k-s j-n}\right. \\
& \left.\times(\log c)^{k-s(j-1)-n} y^{s}\right) \frac{t^{k}}{(k-s j) ! s !} \text {. }
\end{aligned}
$$

Equating the coefficients of like powers of $t$ on both sides of the above equation, yields assertion (3.11).

Theorem 3.8. Let $\mathrm{a}, \mathrm{b}$ and $\mathrm{c}$ be positive integers by $\mathrm{a} \neq \mathrm{b}$. Then for $\mathrm{x}, \mathrm{y} \in \mathbb{R}$ and $\mathrm{n} \geqslant 0$, we have

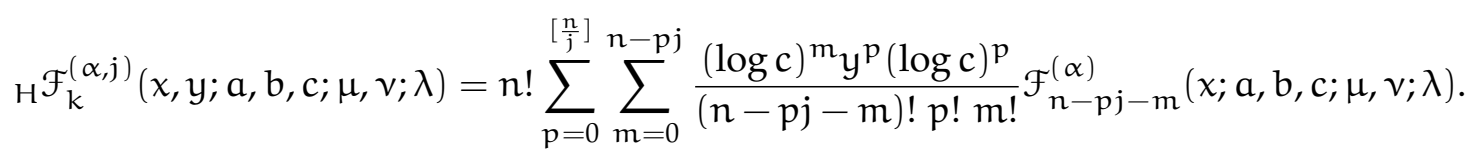

Proof. By the use of equation (1.7) and expansions of $e^{(\log c) t}$ and $e^{(\log c) y t^{j}}$ in generating equation (2.1) with $x \rightarrow x+1$, we have

$$
\sum_{n=0}^{\infty} H_{\mathcal{F}_{n}^{(\alpha, j)}}(x+1, y ; a, b, c ; \mu, v ; \lambda) \frac{\mathrm{t}^{\mathrm{n}}}{n !}=\sum_{n=0}^{\infty} \mathcal{F}_{n}^{(\alpha)}(x ; a, b, c ; \mu, v ; \lambda) \frac{\mathrm{t}^{\mathrm{n}}}{n !} \sum_{m=0}^{\infty}(\log c)^{\mathrm{m}} \frac{\mathrm{t}^{\mathrm{m}}}{\mathrm{m} !} \sum_{p=0}^{\infty} y^{\mathrm{p}}(\log c)^{p} \frac{\mathrm{t}^{p j}}{p^{\mathrm{j}}},
$$

which on applying Cauchy product rule becomes

$$
\begin{aligned}
\sum_{n=0}^{\infty} H_{\mathcal{F}_{n}^{(\alpha, j)}} & (x+1, y ; a, b, c ; \mu, v ; \lambda) \frac{t^{n}}{n !} \\
= & \sum_{n=0}^{\infty}\left(\sum_{m=0}^{n}\left(\begin{array}{c}
n \\
m
\end{array}\right) \mathcal{F}_{n-m}^{(\alpha)}(x ; a, b, c ; \mu, v ; \lambda)(\log c)^{m}\right) \frac{t^{n}}{n !}\left(\sum_{p=0}^{\infty} y^{p}(\log c)^{p} \frac{t^{p j}}{p !}\right) \\
& =\sum_{n=0}^{\infty}\left(\sum_{p=0}^{\left[\frac{n}{j}\right]} \sum_{m=0}^{n-p j}\left(\begin{array}{c}
n-p j \\
m
\end{array}\right) \mathcal{F}_{n-p j-m}^{(\alpha)}(x ; a, b, c ; \mu, v ; \lambda)(\log c)^{m} y^{p}(\log c)^{p}\right) \frac{t^{n}}{(n-p j) ! p !} .
\end{aligned}
$$
(3.12).

Equating the coefficients of like powers of $t$ on both sides of the above equation, yields assertion 
Next, we find multiplication formula for the generalized-Apostol-type-Gould-Hopper polynomials and for their special cases. For this we prove the following results.

Theorem 3.9. Let $\mathrm{a}, \mathrm{b}, \mathrm{c} \in \mathbb{R}^{+}, \mathrm{x}, \mathrm{y} \in \mathbb{R} ; \lambda, \mu, v \in \mathbb{C} ; \mathrm{r} \in \mathbb{N}$ and $\mathrm{n} \in \mathbb{N}_{0}$. The following multiplication formula for the generalized-Apostol-type-Gould-Hopper polynomials holds true:

$$
\begin{aligned}
& H^{\mathcal{F}_{n}^{(\alpha, j)}}\left(r x, r^{j} y ; a, b, \frac{b}{a} ; \mu, v ; \lambda\right)=r^{n-v \alpha} a^{(r-1) \alpha t} \sum_{v_{1}, v_{2}, \ldots, v_{r-1} \geqslant 0}\left(\begin{array}{c}
\alpha \\
v_{1}, v_{2}, \ldots, v_{r-1}
\end{array}\right) \\
& \times(-\lambda)^{m_{H} \mathcal{F}_{n}^{(\alpha, j)}}\left(\left(x+\frac{m}{r}, y ; a, b, \frac{b}{a} ; \mu, v ; \lambda^{r}\right) \text { for } r\right. \text { odd, } \\
& H^{\mathcal{F}_{n}(l, j)}\left(r \chi, r^{j} y ; a, b, \frac{b}{a} ; \mu, v ; \lambda\right)=\frac{(-1)^{l} 2^{\mu l} r^{n-v l}}{(n+1)_{(1-v) l}} a^{(r-1) l t} \sum_{\substack{0 \leqslant v_{1} \leqslant v_{2} \leqslant v_{r-1} \leqslant l \\
v_{1}+v_{2}+\cdots+v_{r-1}=l}}\left(\begin{array}{c}
l \\
v_{1}, v_{2}, \ldots, v_{r-1}
\end{array}\right) \\
& \times(-\lambda)^{m}{ }_{H} \mathfrak{B}_{n+(1-v) l}^{(l, j)}\left(\left(x+\frac{m}{r}, y ; a, b, \frac{b}{a} ; \lambda^{r}\right) \text { for } r\right. \text { even, }
\end{aligned}
$$

where $v_{1}+2 v_{2}+\cdots+(r-1) v_{r-1}=m$.

Proof. We consider generating equation (2.1) in the following form:

$$
\sum_{n=0}^{\infty} H_{\mathcal{F}_{n}^{(\alpha, j)}}\left(r x, r^{j} y ; a, b, \frac{b}{a} ; \mu, v ; \lambda\right) \frac{t^{n}}{n !}=\frac{1}{r^{v \alpha}}\left(\frac{2^{\mu}(r t)^{v}}{\lambda^{r} b^{r t}+a^{r t}}\right)^{\alpha}\left(\frac{\lambda^{r} b^{r t}+a^{r t}}{\lambda b^{t}+a^{t}}\right)^{\alpha}\left(\frac{b}{a}\right)^{r x t+y(r t)^{j}}
$$

which on simplifying becomes

$$
\begin{aligned}
& \sum_{n=0}^{\infty} H_{\mathcal{F}_{n}^{(\alpha, j)}}\left(r x, r^{j} y ; a, b, \frac{b}{a} ; \mu, v ; \lambda\right) \frac{t^{n}}{n !}=\frac{1}{r^{v \alpha}}\left(\frac{2^{\mu}(r t)^{v}}{\lambda^{r} b^{r t}+a^{r t}}\right)^{\alpha} a^{(r-1) \alpha t}\left(\frac{\lambda^{r}\left(\frac{b}{a}\right)^{r t}+1}{\lambda\left(\frac{b}{a}\right)^{t}+1}\right)^{\alpha}\left(\frac{b}{a}\right)^{r x t+y(r t)^{j}} \\
& =\frac{1}{r^{v \alpha}}\left(\frac{2^{\mu}(r t)^{v}}{\lambda^{r} b^{r t}+a^{r t}}\right)^{\alpha} a^{(r-1) \alpha t}\left(\sum_{k=0}^{r-1}\left(-\lambda\left(\frac{b}{a}\right)^{t}\right)^{k}\right)^{\alpha}\left(\frac{b}{a}\right)^{r \times t+y(r t)^{j}} \text {. }
\end{aligned}
$$

Now, on use of the following formula for the generalized multinomial identity [4]:

$$
\left(1+x_{1}+x_{2}+\cdots+x_{m}\right)^{\alpha}=\sum_{v_{1}, v_{2}, \ldots, v_{m} \geqslant 0}\left(\begin{array}{c}
\alpha \\
v_{1}, v_{2}, \ldots, v_{m}
\end{array}\right) x_{1}^{v_{1}} x_{2}^{v_{2}} \cdots x_{m}^{v_{m}}
$$

the above equation becomes

$$
\begin{aligned}
& \sum_{n=0}^{\infty} H_{H} \mathcal{F}_{n}^{(\alpha, j)}\left(r \chi, r^{j} y ; a, b, \frac{b}{a} ; \mu, v ; \lambda\right) \frac{t^{n}}{n !} \\
&=\frac{1}{r^{v \alpha}}\left(\frac{2^{\mu}(r t)^{v}}{\lambda^{r} b^{r t}+a^{r t}}\right)^{\alpha} a^{(r-1) \alpha t} \\
& \quad \times \sum_{v_{1}, v_{2}, \ldots, v_{r-1} \geqslant 0}\left(\begin{array}{c}
\alpha \\
v_{1}, v_{2}, \ldots, v_{r-1}
\end{array}\right)(-\lambda)^{v_{1}+2 v_{2}+\cdots+(r-1) v_{r-1}\left(\left(\frac{b}{a}\right)^{t}\right)^{v_{1}+2 v_{2}+\cdots+(r-1) v_{r-1}}\left(\frac{b}{a}\right)^{r x t+y(r t)^{j}}} \\
&= \frac{1}{r^{v \alpha}}\left(\frac{2^{\mu}(r t)^{v}}{\lambda^{r} b^{r t}+a^{r t}}\right)^{\alpha} a^{(r-1) \alpha t} \sum_{v_{1}, v_{2}, \ldots, v_{r-1} \geqslant 0}\left(\begin{array}{c}
\alpha \\
v_{1}, v_{2}, \ldots, v_{r-1}
\end{array}\right)(-\lambda)^{m}\left(\frac{b}{a}\right)^{\left(x+\frac{m}{r}\right) r t+y(r t)^{j}}
\end{aligned}
$$


Now, using equation (2.1) in above equation, we have

$$
\begin{aligned}
& \sum_{n=0}^{\infty} H_{\mathcal{F}_{n}^{(\alpha, j)}}\left(r \chi, r^{j} y ; a, b, \frac{b}{a} ; \mu, v ; \lambda\right) \frac{t^{n}}{n !}=\sum_{n=0}^{\infty}\left(r^{n-v \alpha} a^{(r-1) \alpha t} \sum_{v_{1}, v_{2}, \ldots, v_{r-1} \geqslant 0}\left(\begin{array}{c}
\alpha \\
v_{1}, v_{2}, \ldots, v_{r-1}
\end{array}\right)\right. \\
& \times(-\lambda)^{m} \sum_{n=0}^{\infty} H_{\mathcal{F}_{n}^{(\alpha, j)}}\left(\left(x+\frac{m}{r}, y ; a, b, \frac{b}{a} ; \mu, v ; \lambda^{r}\right)\right) \frac{t^{n}}{n !} .
\end{aligned}
$$

Equation the coefficients of same power of $t$ on both sides of above equation yields assertion (3.13). Again, with the help of following definition for the multinomial identity [4]:

$$
\left(x_{1}+x_{2}+\cdots+x_{m}\right)^{n}=\sum_{\substack{0 \leqslant v_{1} \leqslant v_{2} \leqslant v_{m} \leqslant n \\
v_{1}+v_{2}+\cdots+v_{m}=n}}\left(\begin{array}{c}
n \\
v_{1}, v_{2}, \ldots, v_{m}
\end{array}\right) x_{1}^{v_{1}} x_{2}^{v_{2}} \cdots x_{m}^{v_{m}}
$$

for $r$ even, we can similarly prove assertion (3.14). The proof is completed.

In view of Remarks 2.3-2.5, we obtain the following consequences of Theorem 3.9.

Corollary 3.10. Let $\mathrm{a}, \mathrm{b}, \mathrm{c} \in \mathbb{R}^{+}, \mathrm{x}, \mathrm{y} \in \mathbb{R} ; \lambda \in \mathbb{C} ; \mathrm{r} \in \mathbb{N}$ and $\mathrm{n} \in \mathbb{N}_{0}$. The following multiplication formula for the generalized-Gould-Hopper-Apostol-Bernoulli polynomials holds true:

$$
\begin{aligned}
& H^{\mathfrak{B}_{n}^{(\alpha, j)}}\left(r \chi, r^{j} y ; a, b, \frac{b}{a} ; \lambda\right)=r^{n-\alpha} a^{(r-1) \alpha t} \sum_{v_{1}, v_{2}, \ldots, v_{r-1} \geqslant 0}\left(\begin{array}{c}
\alpha \\
v_{1}, v_{2}, \ldots, v_{r-1}
\end{array}\right) \\
& \times(-\lambda)^{m}{ }_{H^{B_{n}}}^{(\alpha, j)}\left(\left(x+\frac{m}{r}, y ; a, b, \frac{b}{a} ; \lambda^{r}\right) .\right.
\end{aligned}
$$

Corollary 3.11. Let $\mathrm{a}, \mathrm{b}, \mathrm{c} \in \mathbb{R}^{+}, \mathrm{x}, \mathrm{y} \in \mathbb{R} ; \lambda \in \mathbb{C} ; \mathrm{r} \in \mathbb{N}$ and $\mathrm{n} \in \mathbb{N}_{0}$. The following multiplication formula for the generalized-Gould-Hopper-Apostol-Euler polynomials holds true:

$$
\begin{aligned}
H \mathfrak{E}_{n}^{(\alpha, j)}\left(r x, r^{j} y ; a, b, \frac{b}{a} ; \lambda\right)= & r^{n} a^{(r-1) \alpha t} \sum_{v_{1}, v_{2}, \ldots, v_{r-1} \geqslant 0}\left(\begin{array}{c}
\alpha \\
v_{1}, v_{2}, \ldots, v_{r-1}
\end{array}\right) \\
& \times(-\lambda)^{m}{ }_{H} \mathfrak{E}_{n}^{(\alpha, j)}\left(\left(x+\frac{m}{r}, y ; a, b, \frac{b}{a} ; \lambda^{r}\right) \text { for } r\right. \text { odd, } \\
H \mathfrak{E}_{n}^{(l, j)}\left(r x, r^{j} y ; a, b, \frac{b}{a} ; \lambda\right)= & \frac{(-1)^{l} 2^{l} r^{n}}{(n+1)_{l}} a^{(r-1) l t} \sum_{\substack{0 \leqslant v_{1} \leqslant v_{2} \leqslant v_{r-1} \leqslant l \\
v_{1}+v_{2}+\cdots+v_{r-1}=l}}\left(\begin{array}{c}
l \\
v_{1}, v_{2}, \ldots, v_{r-1}
\end{array}\right) \\
& \times(-\lambda)^{m}{ }_{H} \mathfrak{B}_{n+l}^{(l, j)}\left(\left(x+\frac{m}{r}, y ; a, b, \frac{b}{a} ; \lambda^{r}\right) \text { for } r\right. \text { even. }
\end{aligned}
$$

Corollary 3.12. Let $\mathrm{a}, \mathrm{b}, \mathrm{c} \in \mathbb{R}^{+}, \mathrm{x}, \mathrm{y} \in \mathbb{R} ; \lambda \in \mathbb{C} ; \mathrm{r} \in \mathbb{N}$ and $\mathrm{n} \in \mathbb{N}_{0}$. The following multiplication formula for the generalized-Gould-Hopper-Apostol-Genocchi polynomials holds true:

$$
\begin{aligned}
& H \mathcal{G}_{n}^{(\alpha, j)}\left(r x, r^{j} y ; a, b, \frac{b}{a} ; \lambda\right)=r^{n-\alpha} a^{(r-1) \alpha t} \sum_{v_{1}, v_{2}, \ldots, v_{r-1} \geqslant 0}\left(\begin{array}{c}
\alpha \\
v_{1}, v_{2}, \ldots, v_{r-1}
\end{array}\right) \\
& \times(-\lambda){ }_{H}{ }_{H} \mathcal{G}_{n}^{(\alpha, j)}\left(\left(x+\frac{m}{r}, y ; a, b, \frac{b}{a} ; \lambda^{r}\right) \text { for } r\right. \text { odd, } \\
& H \mathcal{G}_{n}^{(l, j)}\left(r x, r^{j} y ; a, b, \frac{b}{a} ; \lambda\right)=(-1)^{l} 2^{l} r^{n-l} a^{(r-1) l t} \sum_{\substack{0 \leqslant v_{1} \leqslant v_{2} \leqslant v_{r-1} \leqslant l \\
v_{1}+v_{2}+\cdots+v_{r-1}=l}}\left(\begin{array}{c}
l \\
v_{1}, v_{2}, \ldots, v_{r-1}
\end{array}\right) \\
& \times(-\lambda)^{m}{ }_{H} \mathfrak{B}_{\mathfrak{n}}^{(l, j)}\left(\left(x+\frac{m}{r}, y ; a, b, \frac{b}{a} ; \lambda^{r}\right) \text { for } r\right. \text { even. }
\end{aligned}
$$


Further, we derive some explicit formulas involving some known functions. For this we recall the following definition.

Definition 3.13. Let $a, b \in \mathbb{R}^{+}, x \in \mathbb{R}, \lambda \in \mathbb{C}$ and $v \in \mathbb{N}_{0}$. The generalized array type polynomials $\mathcal{S}_{v}^{n}(x ; a, b ; \lambda)$ are defined by [38]:

$$
\frac{\left(\lambda b^{t}-a^{t}\right)^{v}}{v !} b^{x t}=\sum_{n=0}^{\infty} \mathcal{S}_{v}^{n}(x ; a, b ; \lambda) \frac{t^{n}}{n !},
$$

where $\mathcal{S}(n, v ; a, b ; \lambda):=\mathcal{S}_{v}^{n}(0 ; a, b ; \lambda)$ are generalized $\lambda$-Stirling type numbers of second kind.

Definition 3.14. The polynomials $Y_{n}^{v}(x, \lambda ; a)$ are defined by [24, 38]:

$$
\left(\frac{t}{\lambda a^{t}-1}\right)^{v} a^{x t}=\sum_{n=0}^{\infty} Y_{n}^{v}(x, \lambda ; a) \frac{t^{n}}{n !} \quad(a \geqslant 1),
$$

where $Y_{n}^{v}(\lambda ; a):=Y_{n}^{v}(x, \lambda ; a)$ are the corresponding numbers.

Definition 3.15. The generalized Hurwitz-Lerch Zeta function $\Phi_{\mu}(z, s, a)$ [12] is defined by

$$
\Phi_{\mu}(z, s, a)=\sum_{n=0}^{\infty} \frac{(\mu)_{n}}{n !} \frac{z^{n}}{(n+a)^{s}} .
$$

Theorem 3.16. Let $\mathrm{a}, \mathrm{b} \in \mathbb{R}^{+}, x, y \in \mathbb{R} ; \lambda, \mu, v \in \mathbb{C} ; \mathrm{r} \in \mathbb{N}$ and $\mathrm{n} \in \mathbb{N}_{0}$. The following explicit formula for the generalized-Apostol-type-Gould-Hopper polynomials holds true:

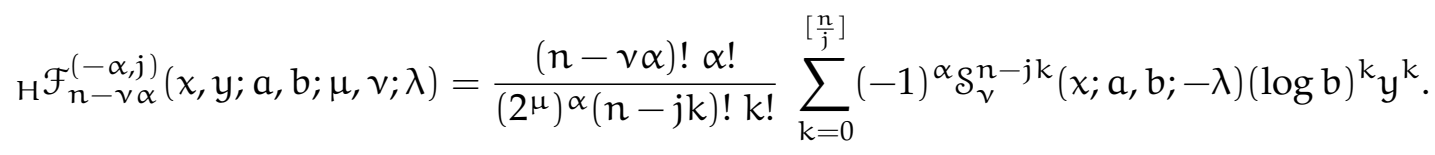

Proof. Replacing $\mathrm{c}$ by $\mathrm{b}$ in generating equation (2.1) yields

$$
\begin{aligned}
& \sum_{n=0}^{\infty} H^{\mathcal{F}_{n}^{(-\alpha, j)}}(x, y ; a, b ; \mu, v ; \lambda) \frac{t^{n}}{n !}=\left(\frac{2^{\mu} t^{v}}{\lambda b^{t}+a^{t}}\right)^{-\alpha} b^{x t+y t^{j}} \\
& =\frac{\alpha !}{\left(2^{\mu} t^{v}\right)^{\alpha}}\left(\frac{\left(\lambda b^{t}+a^{t}\right)^{\alpha}}{\alpha !} b^{x t}\right) b^{y t^{j}} \\
& =\frac{\alpha !}{\left(2^{\mu} t^{v}\right)^{\alpha}} \sum_{n=0}^{\infty} \sum_{k=0}^{\infty}(-1)^{\alpha} \mathcal{S}_{\alpha}^{n}(x ; a, b ;-\lambda)(\log b)^{k} y^{k} \frac{t^{n+j k}}{n ! k !} \\
& =\frac{\alpha !}{\left(2^{\mu} t^{v}\right)^{\alpha}} \sum_{n=0}^{\infty} \sum_{k=0}^{\left[\frac{n}{j}\right]}(-1)^{\alpha} \mathcal{S}_{\alpha}^{n-j k}(x ; a, b ;-\lambda)(\log b)^{k} y^{k} \frac{t^{n}}{(n-j k) ! k !},
\end{aligned}
$$

which on equating the coefficients of same powers of $t$ yields assertion (3.15).

Theorem 3.17. The following explicit formula for the generalized-Apostol-type-Gould-Hopper polynomials holds true:

$$
{ }_{H} \mathcal{F}_{n-v \alpha}^{(-\alpha, j)}(x, y ; a, b ; \mu, v ; \lambda)=\frac{(n-v \alpha) ! \alpha !}{\left(2^{\mu}\right)^{\alpha}} \sum_{k=0}^{n}\left(\begin{array}{l}
n \\
k
\end{array}\right)(-1)^{\alpha} \mathcal{S}(n-k, \alpha ; a, b ;-\lambda) H_{k}^{(j)}(x, y, b) .
$$

Theorem 3.18. Let $\mathrm{a}, \mathrm{b} \in \mathbb{R}^{+}, x, y \in \mathbb{R} ; \lambda, \mu, v \in \mathbb{C} ; \mathrm{r} \in \mathbb{N}$ and $\mathrm{n} \in \mathbb{N}_{0}$. The following explicit formula for the generalized-Apostol-type-Gould-Hopper polynomials holds true:

$$
{ }_{H} \mathcal{F}_{n}^{(\alpha, j)}(x, y ; a, b ; \mu, 1 ; \lambda)=\left(\frac{2^{\mu}}{a^{t}}\right)^{\alpha} \sum_{n=0}^{\infty} \sum_{k=0}^{\left[\frac{n}{j}\right]}(-1)^{\alpha} y_{n-j k}^{\alpha}\left(x ; \frac{b}{a} ;-\lambda\right)(\log b)^{k} y^{k} \frac{t^{n}}{(n-j k) ! k !} .
$$


Proof. Replacing $\mathrm{c}$ by $\mathrm{b}$ in generating equation (2.1) yields

$$
\begin{aligned}
\sum_{n=0}^{\infty} H_{H_{n}} \mathcal{F}^{(\alpha, j)}(x, y ; a, b ; \mu, 1 ; \lambda) \frac{t^{n}}{n !} & =\left(\frac{2^{\mu} t}{\lambda b^{t}+a^{t}}\right)^{\alpha} b^{x t+y t^{j}} \\
& =\left(\frac{2^{\mu}}{a^{t}}\right)^{\alpha}\left(\left(\frac{t}{\lambda\left(\frac{b}{a}\right)^{t}+1}\right)^{\alpha} b^{x t}\right) b^{y t^{j}} \\
& =\left(\frac{2^{\mu}}{a^{t}}\right)^{\alpha} \sum_{n=0}^{\infty} \sum_{k=0}^{\infty}(-1)^{\alpha} y_{n}^{\alpha}\left(x ; \frac{b}{a} ;-\lambda\right)(\log b)^{k} y^{k} \frac{t^{n+j k}}{n ! k !} \\
& =\left(\frac{2^{\mu}}{a^{t}}\right)^{\alpha} \sum_{n=0}^{\infty} \sum_{k=0}^{\left[\frac{n}{j}\right]}(-1)^{\alpha} y_{n-j k}^{\alpha}\left(x ; \frac{b}{a} ;-\lambda\right)(\log b)^{k} y^{k} \frac{t^{n}}{(n-j k) ! k !},
\end{aligned}
$$

which on equating the coefficients of same powers of $t$ yields assertion (3.16).

Theorem 3.19. The following explicit formula for the generalized-Apostol-type-Gould-Hopper polynomials holds true:

$$
{ }_{H} \mathcal{F}_{n}^{(\alpha, j)}(x, y ; a, b ; \mu, 1 ; \lambda)=\left(\frac{2^{\mu}}{a^{t}}\right)^{\alpha} \sum_{k=0}^{n}\left(\begin{array}{l}
n \\
k
\end{array}\right)(-1)^{\alpha} y_{n-k}^{\alpha}\left(\frac{b}{a} ;-\lambda\right) H_{k}^{(j)}(x, y, b) .
$$

Theorem 3.20. Let $\mathrm{a}, \mathrm{b} \in \mathbb{R}^{+}, x, y \in \mathbb{R} ; \lambda, \mu, v \in \mathbb{C} ; \mathrm{r} \in \mathbb{N}$ and $\mathrm{n} \in \mathbb{N}_{0}$. The following explicit formula for the generalized-Apostol-type-Gould-Hopper polynomials holds true:

$$
{ }_{H} \mathcal{F}_{n+v \alpha}^{(\alpha, j)}\left(x, y ; a, b, \frac{b}{a} ; \mu, v ; \lambda\right)=\left(\frac{2^{\mu}}{a^{t}}\right)^{\alpha} \frac{(n+v \alpha) !}{(n-j l) ! l !} \sum_{l=0}^{\left[\frac{n}{j}\right]}\left(\log \frac{b}{a}\right)^{n-j l+l} \Phi_{\alpha}(\lambda,-n+j l, x) y^{l}
$$

Proof. We consider generating function (2.1) in the following form:

$$
\begin{aligned}
\sum_{n=0}^{\infty} H_{H_{n}} \mathcal{F}_{n}^{(\alpha, j)}\left(x, y ; a, b, \frac{b}{a} ; \mu, v ; \lambda\right) \frac{t^{n-v \alpha}}{n !} & =\left(\frac{2^{\mu}}{a^{t}}\right)^{\alpha}\left(\lambda\left(\frac{b}{a}\right)^{t}+1\right)^{-\alpha}\left(\frac{b}{a}\right)^{x t+y t^{j}} \\
& =\left(\frac{2^{\mu}}{a^{t}}\right)^{\alpha} \sum_{k=0}^{\infty} \frac{(\alpha)_{k}}{k !} \lambda^{k}\left(\frac{b}{a}\right)^{k t}\left(\frac{b}{a}\right)^{x t+y t^{j}} \\
& =\left(\frac{2^{\mu}}{a^{t}}\right)^{\alpha} \sum_{n=0}^{\infty} \sum_{k=0}^{\infty} \frac{(\alpha)_{k}}{k !} \lambda^{k}\left(\log \frac{b}{a}\right)^{n}(k+x)^{n} \frac{t^{n}}{n !}\left(\frac{b}{a}\right)^{y t^{j}} \\
& =\left(\frac{2^{\mu}}{a^{t}}\right)^{\alpha} \sum_{n=0}^{\infty}\left(\log \frac{b}{a}\right)^{n+l} \Phi_{\alpha}(\lambda,-n, x) \frac{t^{n}}{n !} \sum_{l=0}^{\infty} y^{l} \frac{t^{j l}}{l !} \\
& =\left(\frac{2^{\mu}}{a^{t}}\right)^{\alpha} \sum_{n=0}^{\infty} \sum_{l=0}^{\left[\frac{n}{j}\right]}\left(\log \frac{b}{a}\right)^{n-j l+l} \Phi_{\alpha}(\lambda,-n+j l, x) y^{l} \frac{t^{n}}{(n-j l) ! l !},
\end{aligned}
$$

which on equating the coefficients of same powers of $t$ in both sides of resultant equation yields assertion (3.17). als.

Next, we establish some symmetric identities for generalized-Apostol-type-Gould-Hopper polynomi- 


\section{Symmetric identities}

In mathematics and statistics, sums of powers occur in a number of contexts. Many combinatorial problems can be solved with the aid of these power sums particularly occur in Euler's sum of powers conjecture, Fermat Catalan conjecture, Lander, Parkin and Selfridge conjectures, Prouhet-Tarry-Escott problem etc.. The power sum symmetric polynomials are considered to be building block for symmetric polynomials.

The symmetry identities related to classical and Apostol type polynomials are established by many authors [18, 20-22, 36]. This provides motivation to establish symmetry identities for the generalizedApostol-type-Gould-Hopper polynomials.

In order to derive the symmetry identities for the $\operatorname{GGdHATP}_{H} \mathcal{F}_{n}^{(\alpha)}(x, y ; a, b, c ; \mu, v ; \lambda)$, we prove the following results.

Theorem 4.1. For all integers $l, m>0$ and $n \geqslant 0 ; \alpha \geqslant 1 ; \lambda, \mu, v \in \mathbb{C}$, and $a, b, c \in \mathbb{R}^{+}$, the following symmetry identity for the generalized-Apostol-type-Gould-Hopper polynomials holds true:

$$
\begin{aligned}
\sum_{j=0}^{n}\left(\begin{array}{c}
n \\
j
\end{array}\right) l^{n-j} m^{j+v}{ }_{H} \mathcal{F}_{n-j}^{(\alpha)}\left(m x, m^{j} y ; a, b, c ; \mu, v ; \lambda\right) \sum_{i=0}^{j}\left(\begin{array}{l}
j \\
i
\end{array}\right) a^{(l-1) m t} \mathcal{S}_{i}(l-1 ;-\lambda) \\
\quad \times{ }_{H} \mathcal{F}_{j-i}^{(\alpha-1)}\left(l X, l^{j} Y ; a, b, c ; \mu, v ; \lambda\right) \\
=\sum_{j=0}^{n}\left(\begin{array}{c}
n \\
j
\end{array}\right) m^{n-j} l^{j+v}{ }_{H} \mathcal{F}_{n-j}^{(\alpha)}\left(l x, l^{j} y ; a, b, c ; \mu, v ; \lambda\right) \sum_{i=0}^{j}\left(\begin{array}{l}
j \\
i
\end{array}\right) a^{(m-1) l t} \mathcal{S}_{i}(m-1 ;-\lambda) \\
\quad \times{ }_{H} \mathcal{F}_{j-i}^{(\alpha-1)}\left(m X, m^{j} Y ; a, b, c ; \mu, v ; \lambda\right) .
\end{aligned}
$$

Proof. Let

$$
G(t):=\frac{\left.2^{\mu(2 \alpha-1)} t^{\nu(2 \alpha-1)} c^{l m x t+y(l m t}\right)^{j}\left(\lambda b^{l m t}+a^{l m t}\right) c^{l m X t+Y(l m t)^{j}}}{\left(\lambda b^{l t}+a^{l t}\right)^{\alpha}\left(\lambda b^{m t}+a^{m t}\right)^{\alpha}},
$$

which on rearranging the powers becomes

$$
\begin{aligned}
G(t)= & \frac{1}{l^{v \alpha} m^{v(\alpha-1)}}\left(\frac{2^{\mu} l^{\nu} t^{\nu}}{\lambda b^{l t}+a^{l t}}\right)^{\alpha} c^{l m x t+y(l m t)^{j}}\left(\frac{\lambda b^{l m t}+a^{l m t}}{\lambda b^{m t}+a^{m t}}\right) \\
& \times\left(\frac{2^{\mu} m^{\nu} t^{\nu}}{\lambda b^{m t}+a^{m t}}\right)^{\alpha-1} c^{l m X t+Y(l m t)^{j}} .
\end{aligned}
$$

Since the expression (4.2) for $G(t)$ is symmetric in $l$ and $m$, therefore we can expand $G(t)$ into series in two ways. First, we consider the following expansion:

$$
G(t)=\frac{1}{l^{v \alpha} m^{v(\alpha-1)}}\left(\frac{2^{\mu}(l t)^{v}}{\lambda b^{l t}+a^{l t}}\right)^{\alpha} c^{l m x t+y(l m t)^{j}}\left(\frac{\lambda b^{l m t}+a^{l m t}}{\lambda b^{m t}+a^{m t}}\right)\left(\frac{2^{\mu}(m t)^{v}}{\lambda b^{m t}+a^{m t}}\right)^{\alpha-1} c^{l m X t+Y(l m t)^{j}},
$$

which on using generating equation (2.1) and the following formula for the numbers $\mathcal{S}_{k}(n ; \lambda)$ :

$$
\frac{\lambda e^{(n+1) t}-1}{\lambda e^{t}-1}=\sum_{k=0}^{\infty} \mathcal{S}_{k}(n ; \lambda) \frac{t^{k}}{k !}
$$

gives

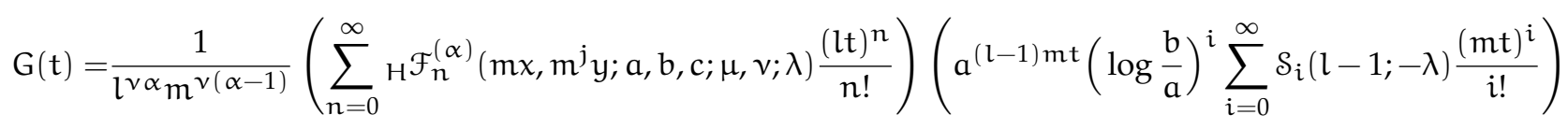




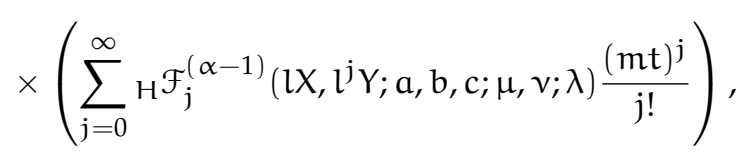

which on using [40, p.890 Corollary 2] and Cauchy product rule gives

$$
\begin{aligned}
G(t)= & \frac{a^{(l-1) m t}}{l^{v \alpha} m^{v \alpha}} \sum_{n=0}^{\infty}\left(\sum_{j=0}^{n}\left(\begin{array}{l}
n \\
j
\end{array}\right) l^{n-j} m^{j+v}{ }_{H} \mathcal{F}_{n-j}^{(\alpha)}\left(m x, m^{j} y ; a, b, c ; \mu, v ; \lambda\right)\right. \\
& \left.\quad \times \sum_{i=0}^{j}\left(\begin{array}{c}
j \\
i
\end{array}\right)\left(\log \frac{b}{a}\right)^{i} \mathcal{S}_{i}(l-1 ;-\lambda)_{H} \mathcal{F}_{j-i}^{(\alpha-1)}\left(l X, l^{j} Y ; a, b, c ; \mu, v ; \lambda\right)\right) \frac{t^{n}}{n !} .
\end{aligned}
$$

Similarly, we have

$$
\begin{aligned}
G(t)= & \frac{a^{(m-1) l t}}{m^{v \alpha} l^{v \alpha}} \sum_{n=0}^{\infty}\left(\sum_{j=0}^{n}\left(\begin{array}{l}
n \\
j
\end{array}\right) m^{n-j} l^{j+v}{ }_{H} \mathcal{F}_{n-j}^{(\alpha)}\left(l x, l^{j} y ; a, b, c ; \mu, v ; \lambda\right)\right. \\
& \left.\quad \times \sum_{i=0}^{j}\left(\begin{array}{l}
j \\
i
\end{array}\right)\left(\log \frac{b}{a}\right)^{i} \mathcal{S}_{i}(m-1 ;-\lambda)_{H} \mathcal{F}_{j-i}^{(\alpha-1)}\left(m X, m^{j} Y ; a, b, c ; \mu, v ; \lambda\right)\right) \frac{t^{n}}{n !} .
\end{aligned}
$$

Equating the coefficients of same powers of $t$ in r.h.s. of expansions (4.3) and (4.4), we are led to assertion (4.1).

Theorem 4.2. For all integers $l, m>0$ and $n \geqslant 0 ; \alpha \geqslant 1 ; \lambda, \mu, v \in \mathbb{C}$ and $a, b \in \mathbb{R}^{+}$, the following symmetry identity for the generalized-Apostol-type-Gould-Hopper polynomials holds true:

$$
\begin{aligned}
& \sum_{p=0}^{n}\left(\begin{array}{l}
n \\
p
\end{array}\right) \sum_{i=0}^{l-1} \sum_{h=0}^{m-1}(-\lambda)^{i+h} l^{p} m^{n-p}{ }_{H} \mathcal{F}_{p}^{(\alpha)}\left(m x+\frac{m}{l} i, m^{j} y ; a, b, \frac{b}{a} ; \mu, v ; \lambda\right) \\
& \quad \times{ }_{H} \mathcal{F}_{n-p}^{(\alpha)}\left(l X+\frac{l}{m} h, l^{j} Y ; a, b, \frac{b}{a} ; \mu, v ; \lambda\right) \\
& =\sum_{p=0}^{n}\left(\begin{array}{c}
n \\
p
\end{array}\right) \sum_{i=0}^{m-1} \sum_{h=0}^{l-1}(-\lambda)^{i+h} m^{p} l^{n-p}{ }_{H} \mathcal{F}_{p}^{(\alpha)}\left(l x+\frac{l}{m} i, l^{j} y ; a, b, \frac{b}{a} ; \mu, v ; \lambda\right) \\
& \quad \times{ }_{H} \mathcal{F}_{n-p}^{(\alpha)}\left(m X+\frac{m}{l} h, m^{j} Y ; a, b, \frac{b}{a} ; \mu, v ; \lambda\right) .
\end{aligned}
$$

Proof. Let

$$
H(t):=\frac{\left.\left.2^{2 \mu \alpha \alpha} t^{2 v \alpha} \frac{b}{a}^{l m x t+y(l m t}\right)^{j}\left(\lambda^{l} b^{l m t}+a^{l m t}\right)\left(\lambda^{m} b^{l m t}+a^{l m t}\right) \frac{b}{a}^{l m X t+\gamma(l m t}\right)^{j}}{\left(\lambda b^{l t}+a^{l t}\right)^{\alpha+1}\left(\lambda b^{m t}+a^{m t}\right)^{\alpha+1}},
$$

which on rearranging the powers becomes

$$
\begin{aligned}
H(t):= & \frac{1}{l^{v \alpha}} \frac{1}{m^{v \alpha}}\left(\frac{2^{\mu} l^{v} t^{v}}{\lambda b^{l t}+a^{l t}}\right)^{\alpha} \frac{b^{l m x t+y(l m t)^{j}}}{a}\left(\frac{\lambda^{l} b^{l m t}+a^{l m t}}{\lambda b^{m t}+a^{m t}}\right)\left(\frac{2^{\mu} m^{v} t^{v}}{\lambda b^{m t}+a^{m t}}\right)^{\alpha} \\
& \times \frac{b^{l m X t+Y(l m t)^{j}}}{a}\left(\frac{\lambda^{m} b^{l m t}+a^{l m t}}{\lambda b^{l t}+a^{l t}}\right) .
\end{aligned}
$$

Since expression (4.6) for $H(t)$ is symmetric in $l$ and $m$, therefore we can expand $H(t)$ into series in two ways. First, we consider the following expansion:

$$
\begin{aligned}
H(t):= & \frac{1}{l^{v \alpha}} \frac{1}{m^{v \alpha}}\left(\frac{2^{\mu}(l t)^{v}}{\lambda b^{l t}+a^{l t}}\right)^{\alpha} \frac{b}{a}^{l m x t+y(l m t)^{j}}\left(\frac{\lambda^{l} b^{l m t}+a^{l m t}}{\lambda b^{m t}+a^{m t}}\right)\left(\frac{2^{\mu}(m t)^{v}}{\lambda b^{m t}+a^{m t}}\right)^{\alpha} \\
& \times \frac{b^{l m X t+Y(l m t)^{j}}}{a}\left(\frac{\lambda^{m} b^{l m t}+a^{l m t}}{\lambda b^{l t}+a^{l t}}\right) .
\end{aligned}
$$


Now, using the series expansions for $\left(\frac{\lambda^{\mathrm{l}} b^{l m t}+a^{l m t}}{\lambda b^{m t}+a^{m t}}\right)$ and $\left(\frac{\lambda^{m} b^{l m t}+a^{l m t}}{\lambda b^{l t}+a^{l t}}\right)$ in the r.h.s. of equation (4.7), we have

$$
\begin{aligned}
H(t):= & \frac{1}{l^{v \alpha}} \frac{1}{m^{v \alpha}}\left(\frac{2^{\mu}(l t)^{v}}{\lambda b^{l t}+a^{l t}}\right)^{\alpha} \frac{b}{a}^{l m \times t+y(l m t)^{j}}\left(a^{(l-1) m t} \sum_{i=0}^{l-1}(-\lambda)^{i}\left(\frac{b}{a}\right)^{m t i}\right) \\
& \times\left(\frac{2^{\mu}(m t)^{v}}{\lambda b^{m t}+a^{m t}}\right)^{\alpha} \frac{b}{a}^{l m X t+Y(l m t)^{j}}\left(a^{(m-1) l t} \sum_{h=0}^{m-1}(-\lambda)^{h}\left(\frac{b}{a}\right)^{l t h}\right) .
\end{aligned}
$$

Combining the terms in the above equation, we have

$$
\begin{aligned}
& H(t):=\frac{1}{l^{v \alpha}} \frac{1}{m^{v \alpha}} a^{2 m l t-m t-l t} \sum_{i=0}^{l-1}(-\lambda)^{i}\left(\frac{2^{\mu}(l t)^{v}}{\lambda b^{l t}+a^{l t}}\right)^{\alpha} \frac{b}{a}^{\left(m x+\frac{m}{l} i\right) l t+m^{j} y(l t)^{j}} \\
& \times \sum_{h=0}^{m-1}(-\lambda)^{h}\left(\frac{2^{\mu}(m t)^{v}}{\lambda b^{m t}+a^{m t}}\right)^{\alpha} \frac{b}{a}^{\left(l X+\frac{l}{m} h\right) m t+l^{j} Y(m t)^{j}},
\end{aligned}
$$

which on using equation (2.1) becomes

$$
\begin{aligned}
H(t):= & \frac{1}{l^{v \alpha}} \frac{1}{m^{v \alpha}} a^{2 m l t-m t-l t}\left(\sum_{i=0}^{l-1}(-\lambda)^{i} \sum_{p=0}^{\infty} H_{\mathcal{p}} \mathcal{F}^{(\alpha)}\left(m x+\frac{m}{l} i, m^{j} y ; a, b, \frac{b}{a} ; \mu, v ; \lambda\right) \frac{(l t)^{p}}{p !}\right) \\
& \times\left(\sum_{h=0}^{m-1}(-\lambda)^{h} \sum_{n=0}^{\infty} H_{\mathcal{F}_{n}^{(\alpha)}}\left(l X+\frac{l}{m} h, l^{j} Y ; a, b, \frac{b}{a} ; \mu, v ; \lambda\right) \frac{(m t)^{n}}{n !}\right),
\end{aligned}
$$

Applying the Cauchy product rule in the r.h.s. of equation (4.8), we find

$$
\begin{aligned}
H(t):= & \frac{1}{l^{v \alpha}} \frac{1}{m^{v \alpha}} a^{2 m l t-m t-l t} \sum_{n=0}^{\infty} \sum_{p=0}^{n}\left(\begin{array}{l}
n \\
p
\end{array}\right) \sum_{i=0}^{l-1} \sum_{h=0}^{m-1}(-\lambda)^{i+h} l^{p} m^{n-p} \mathcal{F}_{p}^{(\alpha)} \\
& \times\left(m x+\frac{m}{l} i, m^{j} y ; a, b, \frac{b}{a} ; \mu, v ; \lambda\right)_{H} \mathcal{F}_{n-p}^{(\alpha)}\left(l X+\frac{l}{m} h, l^{j} Y ; a, b, \frac{b}{a} ; \mu, v ; \lambda\right) \frac{t^{n}}{n !} .
\end{aligned}
$$

Using the similar way, we obtain the second expansion of $\mathrm{H}(\mathrm{t})$ as:

$$
\begin{aligned}
H(t):= & \frac{1}{m^{v \alpha}} \frac{1}{l^{v \alpha}} a^{2 m l t-m t-l t} \sum_{n=0}^{\infty} \sum_{p=0}^{n}\left(\begin{array}{l}
n \\
p
\end{array}\right) \sum_{i=0}^{m-1} \sum_{h=0}^{l-1}(-\lambda)^{i+h} m^{p} l^{n-p}{ }_{H} \mathcal{F}_{p}^{(\alpha)} \\
& \times\left(l x+\frac{l}{m} i, l^{j} y ; a, b, \frac{b}{a} ; \mu, v ; \lambda\right)_{H} \mathcal{F}_{n-p}^{(\alpha)}\left(m X+\frac{m}{l} h, m^{j} Y ; a, b, \frac{b}{a} ; \mu, v ; \lambda\right) \frac{t^{n}}{n !} .
\end{aligned}
$$

Equating the coefficients of like powers of $t$ in r.h.s. of expansions (4.9) and (4.10), we are led to assertion (4.5).

In view of Remarks 2.3-2.5, we can obtain the symmetry identities for the generalized-Gould-HopperApostol-Bernoulli, Euler and Genocchi polynomials simply by choosing suitable values of parameters $\mu$ and $v$. For the lack of space, we omit them.

\section{Appendix A.}

In 2006, Luo and Srivastava [33] introduced the Milne-Thomson's polynomials $\Phi_{n}^{(\alpha)}(x)$ of single variable defined by

$$
\sum_{n=0}^{\infty} \Phi_{n}^{(\alpha)}(x) \frac{t^{n}}{n !}=f(t, \alpha) e^{x t}
$$


and framed Apostol-Bernoulli and Euler polynomials within the context of Milne-Thomson's polynomials $\Phi_{n}^{(\alpha)}(x)$.

Recently, Dere and Simsek [10] gave a new class of the Milne-Thomson's polynomials $\Phi_{n}^{(\alpha)}(x, y)$ defined by

$$
\sum_{n=0}^{\infty} \Phi_{n}^{(\alpha)}(x, y) \frac{t^{n}}{n !}=f(t, \alpha) e^{x t+h(t, y)} .
$$

In view of equation (A.1), we gave a unified form of generalized Milne-Thomson's polynomials $\Phi_{\mathfrak{n}}^{\alpha}(x, y ; \mu, v ; \lambda)$ defined by

$$
\sum_{n=0}^{\infty} \Phi_{n}^{(\alpha)}(x, y ; \mu, v ; \lambda) \frac{t^{n}}{n !}=f(t, \alpha ; \mu, v ; \lambda) c^{x t+h(t, y)}
$$

Simply by choosing $f(t, \alpha ; \mu, v ; \lambda)=\left(\frac{2^{\mu} l^{v}}{\lambda b^{t}+a^{t}}\right)^{\alpha}$ and $h(t, y)=y t^{j}$, we get generalized-Apostol-typeGould-Hopper polynomials.

\section{References}

[1] P. Appell, J. Kampé de Fériet, Fonctions Hypergéométriques et Hypersphériques: Polynômes d'Hermite, Gauthier-Villars, Paris, (1926). 1.1

[2] S. Araci, M. Acikgoz, A note on the Frobenius-Euler numbers and polynomials associated with Bernstein polynomials, Adv. Stud. Contemp. Math. (Kyungshang), 22 (2012), 399-406. 1

[3] S. Araci, M. Acikgoz, On the von Staudt-Clausen's theorem related to q-Frobenius-Euler numbers, J. Number Theory, 159 (2016), 329-339. 1

[4] L. Comtet, Advanced Combinatorics: The Art of Finite and Infinite Expansions (Translated from the French by J. W. Nienhuys), D. Reidel Publishing Company, Boston-Dordrecht, (1974). 3

[5] G. Dattoli, Hermite-Bessel and Laguerre-Bessel functions: a by-product of the monomiality principle, Advanced Special functions and applications, Procedings of the 1999 Melfi Workshop on generalised special functions and applications, 1999 (1999), 147-164. 1

[6] G. Dattoli, Generalized polynomials, operational identities and their applications, J. Comput. Appl. Math., 118 (2000), 111-123. 1

[7] G. Dattoli, Summation formulae of special functions and multivariable Hermite polynomials, Nuovo Cimento Soc. Ital. Fis. B, 119 (2004), 479-488. 3

[8] G. Dattoli, C. Chiccoli, S. Lorenzutta, G. Maino, A. Torre, Generalized Bessel functions and generalized Hermite polynomials, J. Math. Anal. Appl., 178 (1993), 509-516. 1

[9] G. Dattoli, S. Lorenzutta, G. Maino, A. Torre, C. Cesarano, Generalized Hermite polynomials and super-Gaussian forms, J. Math. Anal. Appl., 203 (1996), 597-609. 1

[10] R. Dere, Y. Simsek, Bernoulli type polynomials on umbral algebra, Russ. J. Math. Phys, 22 (2015), 1-5. Appendix A

[11] H. W. Gould, A. T. Hopper, Operational formulas connected with two generalization of Hermite polynomials, Duke Math. J., 29 (1962), 51-63. 1.2

[12] S. P. Goyal, R. K. Laddha, On the generalized Riemann zeta functions and the generalized Lambert transform, Ganita Sandesh, 11 (1997), 99-108. 3.15

[13] Y. He, S. Araci, H. M. Srivastava, M. Acikgoz, Some new identities for the Apostol-Bernoulli polynomials and the Apostol-Genocchi polynomials, Appl. Math. Comput., 262 (2015), 31-41. 1

[14] H. Jolany, H. Sharifi, R. E. Alikelaye, Some results for the Apostol Genocchi polynomials of higher order, Bull. Malays. Math. Sci. Soc. (2), 36 (2013), 465-479. 1, 1.8

[15] S. Khan, M. A. Pathan, N. A. M. H. Makhboul, G. Yasmin, Implicit summation formula for Hermite and related polynomials, J. Math. Anal. Appl., 344 (2008), 408-416. 3

[16] S. Khan, M. Riyasat, A determinantal approach to Sheffer-Appell polynomials via monomiality principle, J. Math. Anal. Appl., 421 (2015), 806-829. 2

[17] S. Khan, M. Riyasat, Determinantal approach to certain mixed special polynomials related to Gould-Hopper polynomials, Appl. Math. Comput., 251 (2015), 599-614. 2

[18] S. Khan, M. Riyasat, G. Yasmin, Finding symmetry identities for the 2-variable Apostol type polynomials, Tbilisi Math. J., 10 (2017), 65-81. 4

[19] S. Khan, G. Yasmin, M. Riyasat, Certain results for the 2-variable Apostol type and related polynomials, Comput. Math. Appl., 69 (2015), 1367-1382. 1, 2, 2

[20] V. Kurt, Some symmetry identities for the Apostol-type polynomials related to multiple alternating sums, Adv. Difference Equ., 2013 (2013), 32 pages. 4 
[21] V. Kurt, On the Unified Family of Generalized Apostol-type Polynomials of Higher order and Multiple Power Sums, Filomat, 30 (2016), 929-935.

[22] V. Kurt, Some symmetry identities for the unified Apostol-type polynomials and multiple power sums, Filomat, 30 (2016), 583-592. 4

[23] B. Kurt, Y. Simsek, Frobenius-Euler type polynomials related to Hermite-Bernoulli polyomials, AIP Conference Proceedings, 2011 (2011), 385-388. 1

[24] B. Kurt, Y. Simsek, On the generalized Apostol-type Frobenius-Euler polynomials, Adv. Difference Equ., 2013 (2013), 9 pages. $1,3.14$

[25] Q.-M. Luo, Apostol-Euler polynomials of higher order and the Gaussian hypergeometric function, Taiwanese J. Math., 10 (2006), 917-925. 1

[26] Q.-M. Luo, The multiplication formulas for the Apostol-Bernoulli and Apostol-Euler polynomials of higher order, Integral Transforms Spec. Funct., 20 (2009), 377-391. 1

[27] Q.-M. Luo, Extension for the Genocchi polynomials and its Fourier expansions and integral representations, Osaka J. Math., 48 (2011), 291-309. 1

[28] Q.-M. Luo, Multiplication formulas for Apostol-type polynomials and multiple alternating sums, Math. Notes, 91 (2012), 46-57. 1

[29] Q.-M. Luo, The multiplication formulas for the Apostol-Genocchi polynomials, Util. Math., 89 (2012), 179-191. 1

[30] Q.-M. Luo, B.-N. Guo, F. Qi, L. Debnath, Generalization of Bernoulli numbers and polynomials, Int. J. Math. Sci., 59 (2003), 3769-3776. 1

[31] Q.-M. Luo, F. Qi, L. Debnath, Generalization of Euler numbers and polynomials, Int. J. Math. Sci., 61 (2003), $3893-3901$. 1

[32] Q.-M. Luo, H. M. Srivastava, Some generalizations of the Apostol-Bernoulli and Apostol-Euler polynomials, J. Math. Anal. Appl., 308 (2005), 290-302. 1

[33] Q.-M. Luo, H. M. Srivastava, Some relationships between the Apostol-Bernoulli and Apostol-Euler polynomials, Comput. Math. Appl., 51 (2006), 631-642. Appendix A

[34] Q.-M. Luo, H. M. Srivastava, Some generalizations of the Apostol-Genocchi polynomials and the Stirling numbers of the second kind, Appl. Math. Comput., 217 (2011), 5702-5728. 1, 1

[35] M. A. Özarslan, Unified Apostol-Bernoulli, Euler and Genocchi polynomials, Comput. Math. Appl., 62 (2011), $2452-$ 2462. 1

[36] M. A. Özarslan, Hermite-based unified Apostol-Bernoulli, Euler and Genocchi polynomials, Adv. Difference Equ., 2013 (2013), 13 pages. 4

[37] M. A. Pathan, W. A. Khan, Some implicit summation formulas and symmetric identities for the generalized Hermite based-polynomials, Acta Univ. Apulensis Math. Inform., 39 (2014), 113-136. 1.3, 1, 2

[38] Y. Simsek, Generating functions for generalized Stirling type numbers, array type polynomials, Eulerian type polynomials and their applications, Fixed Point Theory Appl., 2013 (2013), 28 pages. 1, 3.13, 3.14

[39] H. M. Srivastava, M. Garg, S. Choudhary, A new generalization of the Bernoulli and related polynomials, Russ. J. Math. Phys., 17 (2010), 251-261. 1, 1.6

[40] H. M. Srivastava, M. A. Özarslan, C. Kaanoğlu, Some families of generating functions for a certain class of three-variable polynomials, Integral Transforms Spec. Funct., 21 (2010), 885-896. 4

[41] B. Yilmaz, M. A. Özarslan, Differential equations for the extended 2D Bernoulli and Euler polynomials, Adv. Difference Equ., 2013 (2013), 16 pages. 1.4, 1, 2 